\title{
SDF1-CXCR4 signaling contributes to persistent pain and hypersensitivity via regulating excitability of primary nociceptive neurons: involvement of ERK-dependent Nav1.8 up-regulation
}

Fei Yang ${ }^{1+}$, Wei Sun ${ }^{1,2+}$, Yan Yang ${ }^{3}$, Yan Wang ${ }^{1,2}$, Chun-Li Li ${ }^{1,2}$, Han Fu ${ }^{1,2}$, Xiao-Liang Wang ${ }^{1,2}$, Fan Yang ${ }^{3}$, Ting $\mathrm{He}^{1,2}$ and Jun Chen ${ }^{1,2,3^{*}}$

\begin{abstract}
Background: Pain is one critical hallmark of inflammatory responses. A large number of studies have demonstrated that stromal cell-derived factor 1 (SDF1, also named as CXCL12) and its cognate receptor C-X-C chemokine receptor type 4 (CXCR4) play an important role in immune reaction and inflammatory processes. However, whether and how SDF1-CXCR4 signaling is involved in inflammatory pain remains unclear.

Methods: Under the intraplantar (i.pl.) bee venom (BV) injection-induced persistent inflammatory pain state, the changes of SDF1 and CXCR4 expression and cellular localization in the rat dorsal root ganglion (DRG) were detected by immunofluorescent staining. The role of SDF1 and CXCR4 in the hyperexcitability of primary nociceptor neurons was assessed by electrophysiological recording. Western blot analysis was used to quantify the DRG Nav1.8 and phosphorylation of ERK (pERK) expression. Behavioral tests were conducted to evaluate the roles of CXCR4 as well as extracellular signal-regulated kinase (ERK) and Nav1.8 in the BV-induced persistent pain and hypersensitivity.

Results: We showed that both SDF1 and CXCR4 were dramatically up-regulated in the DRG in i.pl. BV-induced inflammatory pain model. Double immunofluorescent staining showed that CXCR4 was localized in all sizes (large, medium, and small) of DRG neuronal soma, while SDF1 was exclusively expressed in satellite glial cells (SGCs). Electrophysiological recording showed that bath application with AMD3100, a potent and selective CXCR4 inhibitor, could reverse the hyperexcitability of medium- and small-sized DRG neurons harvested from rats following i.pl. BV injection. Furthermore, we demonstrated that the BV-induced ERK activation and Nav1.8 up-regulation in the DRG could be blocked by pre-antagonism against CXCR4 in the periphery with AMD3100 as well as by blockade of ERK activation by intrathecal (i.t.) or intraplantar (i.pl.) U0126. At behavioral level, the BV-induced persistent spontaneous pain as well as primary mechanical and thermal hypersensitivity could also be significantly suppressed by blocking CXCR4 and Nav1.8 in the periphery as well as by inhibition of ERK activation at the DRG level.

(Continued on next page)
\end{abstract}

\footnotetext{
* Correspondence: junchen@fmmu.edu.cn

${ }^{\dagger}$ Equal contributors

'Institute for Biomedical Sciences of Pain, Tangdu Hospital, The Fourth

Military Medical University, \#569 Xinsi Road, Baqiao, Xi'an 710038, People's

Republic of China

2Key Laboratory of Brain Stress and Behavior, PLA, Xi'an 710038, People's

Republic of China

Full list of author information is available at the end of the article
}

\section{Ciomed Central}

(c) 2015 Yang et al. Open Access This article is distributed under the terms of the Creative Commons Attribution 4.0 International License (http://creativecommons.org/licenses/by/4.0/), which permits unrestricted use, distribution, and reproduction in any medium, provided you give appropriate credit to the original author(s) and the source, provide a link to the Creative Commons license, and indicate if changes were made. The Creative Commons Public Domain Dedication waiver (http://creativecommons.org/publicdomain/zero/1.0/) applies to the data made available in this article, unless otherwise stated. 
(Continued from previous page)

Conclusions: The present results suggest that peripheral inflammatory pain state can trigger over release of SDF1 from the activated SGCS in the DRG by which SGC-neuronal cross-talk is mediated by SDF1-CXCR4 coupling that result in subsequent ERK-dependent Nav1.8 up-regulation, leading to hyperexcitability of tonic type of the primary nociceptor cells and development and maintenance of persistent spontaneous pain and hypersensitivity.

Keywords: Inflammatory pain, Chemokines, SDF1, CXCR4, Nav1.8, Dorsal root ganglion, ERK signaling

\section{Background}

Persistent pain that results from inflammation is a major public health problem worldwide. An increasing number of evidence has demonstrated that the up-regulation of inflammatory mediators, including cytokines and chemokines, is involved in the generation of neuronal hyperexcitability and thus contributes to inflammatory pain and hyperalgesia [1-6].

Chemokines could be sorted into four families: $\mathrm{C}$ family, CC family, CXC family, and CX3C family [7]. Stromal cell-derived factor 1 (SDF1, also named as CXCL12), as a member of the CXC family, is constitutively expressed in various kinds of cells in the peripheral and central nervous system $[8,9]$. Through activating the $\mathrm{C}-\mathrm{X}-\mathrm{C}$ chemokine receptor type 4 (CXCR4), a seven transmembrane Gprotein-coupled receptor, SDF1 exerts multiple biological functions [9]. In addition to the well-established role in the immune system and inflammatory reaction, emerging data have shown that SDF1/CXCR4 system is also involved in neurogenesis, neuronal migration, and neuronal differentiation during the development of the nervous system [10-14]. Recently, the pro-algesic effects of SDF1/ CXCR4 have also been implicated in several pain models. Immunohistochemical studies found that the expression level of SDF1 and CXCR4 was changed in the dorsal root ganglion (DRG) cells in the unilateral sciatic nerve injury (CCI)-induced pain model and the up-regulation of CXCR4 lasted at least for 2 weeks [15]. In the spinal cord injury-induced central neuropathic pain model, KnerlichLukoschus and colleagues demonstrated that SDF1 and CXCR4 expression was continuously increased from 2 to 42 days at the spinal cord level [16]. Moreover, by mapping the cellular and subcellular localization of SDF1 and CXCR4, Reaux-Le and colleagues detailedly reported that SDF1/CXCR4 system was closely related to the nociceptive pathway, especially in the primary nociceptive neurons, and they also found that activating the CXCR4 by intrathecal (i.t.) SDF1 injection could induce mechanical allodynia for 3 days, which could be prevented by the CXCR4-neutralizing antibody [8]. However, to date, the underlying mechanisms of SDF1/CXCR4 involved in the chronic and persistent pain remain unclear.

Recently, in vitro electrophysiological experiments have verified that SDF1 could directly modulate the excitability and firing pattern of neuronal cells through
CXCR4 [17-21]. As for neurons, voltage-gated sodium channels (VGSCs) are the key mediators in cellular excitability and are essential for the generation and propagation of action potentials (AP) [22, 23]. Among all the VGSCs, tetrodotoxin-resistant (TTX-R) sodium channel Nav1.8 and/or Nav1.9 mostly contributes to the enhanced excitability and produces majority of current during AP upstroke [24]. Accumulating data showed that peripheral inflammation and nerve injury could induce up-regulation of Nav1.8 in the medium- and small-sized DRG neurons [25-29]. Furthermore, several studies observed that inflammatory mediators (including TNF- $\alpha$, CCL2, and CXCL1) could directly induce Nav1.8 up-regulation in the DRG and thus excite the primary nociceptive neurons [26, 30,31]. Taking all these data into account, we hypothesized that the above modulating effect of the SDF1/CXCR4 system on the neuronal excitability was in part due to the regulation of Nav1.8 which in turn contributes to the generation of pain.

Subcutaneous intraplantar (i.pl.) injection of bee venom (BV) is a frequently used inflammatory pain model which is suitable for exploring the pathophysiological mechanism of persistent pain and inflammation [32-34]. Thus, in the present study, we firstly examined the expression of CXCR4 and SDF1 in DRG cells under inflammatory pain condition induced by i.pl. BV injection. Then, we investigated the role of CXCR4 in the neuronal excitability and Nav1.8 modulation by applying the selective inhibitor of CXCR4, AMD3100. Finally, we investigated whether blocking CXCR4 could alleviate the inflammatory painrelated behaviors.

\section{Methods}

\section{Animals}

The experiments were performed on male SpragueDawley rats weighing from 180 to $220 \mathrm{~g}$ (purchased from the Laboratory Animal Center of Fourth Military Medical University, FMMU, Xi'an, People's Republic of China). The animals were housed in plastic boxes with access to water and food ad libitum and maintained on a 12-h light/dark cycle (with the lights on at 8:00 a.m. to 8:00 p.m.) at room temperature $\left(22-26{ }^{\circ} \mathrm{C}\right)$. Behavioral evaluations were carried out between 9:00 and 18:30. The rats were acclimated to test boxes for at least 30 min each day for 5 days before testing. The present 
experiment protocols were approved by the Institutional Animal Care and Use Committee of FMMU, and were in accordance with the National Institutes of Health Guide for the Care and Use of Laboratory Animals (NIH Publications No. 80-23) revised 1996. International Association for the Study of Pain (IASP)'s ethical guidelines for pain research in conscious animals was followed. The number of animals used and their suffering were greatly minimized.

\section{Behavioral testing}

\section{Persistent spontaneous nociceptive behavior}

The method to estimate persistent spontaneous nociception (PSN) was based on our previously reports [34]. A $30 \times 30 \times 30 \mathrm{~cm}$ transparent Plexiglas box was placed on a supporting frame of $30 \mathrm{~cm}$ high above the experimental table. The rat was placed in the test box for at least 30 min before administration of any chemical agents. After the acclimation period, an i.pl. injection of BV was made into the center of the plantar surface of one hind paw with slight restraint. BV-induced persistent spontaneous nociception was expressed as the number of paw finches occurring at each 5-min interval for $1 \mathrm{~h}$ following i.pl. BV injection.

\section{Mechanical pain sensitivity}

For examination of mechanical pain sensitivity, the mechanical stimuli were applied by using ascending graded individual von Frey monofilaments with bending forces of $0.8,2.0,4.0,6.0,8.0,10.0,12.0,14.0,16.0,18.0$, $20.0,25.0,30.0,45.0$, and $60 \mathrm{~g}$. The rats were placed on a metal mesh floor covered with plastic box, and von Frey filaments were applied from underneath the metal mesh floor to the plantar area of the bilateral hind paws $1 \mathrm{~h}$ before and $2 \mathrm{~h}$ after i.pl. BV injection. Each von Frey filament was applied ten times (once every several seconds) in order to induce the withdrawal reflex. The bending force value of the von Frey filament that caused an appropriate $50 \%$ occurrence of paw withdrawal was expressed as the paw withdrawal mechanical threshold (PWMT, g). For details, see [32, 34].

\section{Thermal pain sensitivity}

The thermal sensitivity was determined by measuring the withdrawal latency of the hind paws in response to radiant heat [34]. Rats were placed in a plastic chamber on the surface of a 2-mm thick glass plate and the sensitivity to heat stimuli by a TC-1 radiant heat stimulator (new generation of RTY-3 made in Xi'an Bobang Technologies of Chemical Industry Co. Ltd., China, $10 \mathrm{~V}$ ) at $30 \mathrm{~min}$ before and $3 \mathrm{~h}$ after i.pl. BV treatment was measured. The heat stimuli were applied to both the injection site and the corresponding area of the contralateral paw, and the latency was determined as the duration from the beginning of heat stimuli to the occurrence of a marked withdrawal reflex. Five stimuli were repeated for each site, and the latter three values were averaged as mean paw withdrawal thermal latency (PWTL, s). A maximal cutoff of $30 \mathrm{~s}$ was used to avoid excessive tissue injury. The inter-stimulus interval for each heat test was more than $15 \mathrm{~min}$ at the same region and $10 \mathrm{~min}$ at the different paws.

\section{Drug}

A volume of $50 \mu \mathrm{l} \mathrm{BV}$ solution $(4 \mu \mathrm{g} / \mu \mathrm{l}$, Floret Ltd. and its partner company New Techniques Laboratory Ltd., Tbilisi, Georgia, dissolved in $0.9 \%$ sterile saline) was used during the whole experiment. To evaluate the role of CXCR4 in the PSN, thermal, and mechanical pain sensitivity, $\operatorname{AMD} 3100(10,100$, or $200 \mu \mathrm{g} / 20 \mu \mathrm{l}$, Sigma) or vehicle was administered through subcutaneous i.pl. injection 10 min prior to BV treatment. We chose this route of delivery to exclude the spinal roles of CXCR4 in the BV-induced pathological pain processing. To evaluate the role of extracellular signal-regulated kinase (ERK), an isoform of mitogen-activated protein kinase (MAPK), U0126 (Sigma, $10 \mu \mathrm{g}$ dissolved in $10 \mu \mathrm{l}$ DMSO), which was decided according to our previous study $[35,36]$, was administered through intrathecal (i.t.) or intraplantar (i.pl.) injection 10 min prior to BV treatment. Considering the effective duration and peak time, A-803467 (Abcam, $500 \mu \mathrm{g} / 50 \mu \mathrm{l}$ dissolved in DMSO), a selective Nav1.8 blocker, was administered through subcutaneous injection $10 \mathrm{~min}$ prior to or $90 \mathrm{~min}$ post-BV treatment $[37,38]$.

\section{Implantation of intrathecal catheters and administration of drug}

Intrathecal catheter implantation was performed according to the method described previously [39]. Briefly, under sodium pentobarbital anesthesia $(40 \mathrm{mg} / \mathrm{kg}$, i.p.), a sterile polyethylene (PE-10) tube filled with $0.9 \%$ sterile saline was inserted into the L5/L6 intervertebral space, and the tip of the tube was placed at the spinal lumbar enlargement level. The tube was fixed by suturing into the superficial muscle. Then, a tunnel under the skin was made and the tube was pulled out of another skin incision at the neck area where the tube was fixed on the skin. The outer end of the catheter was sealed by melting. The rats were allowed to recover for 3 days in individual cages, and only those without motor disturbance and other neurological deficits were included for further experiments. Drugs or vehicle were administered in volumes of $10 \mu \mathrm{l}$ by microinjection syringe followed by a flush of $10 \mu \mathrm{l}$ saline to ensure drugs were delivered into the subarachnoid space. After drug injection, the outer end of the catheter was sealed again by heat melting. 


\section{Immunohistochemistry}

The rats were anesthetized with $1 \%$ pentobarbital sodium $(50 \mathrm{mg} / \mathrm{kg}$, i.p.), then perfused with physiological saline, followed by $4 \%$ paraformaldehyde in $0.1 \mathrm{M} \mathrm{PB}$ solution. After perfusion, the L4-6 DRGs were removed and postfixed in the same $4 \%$ fixative overnight at $4{ }^{\circ} \mathrm{C}$ and cryoprotected by immersion in $30 \%$ sucrose in $0.1 \mathrm{M} \mathrm{PB}$ at $4{ }^{\circ} \mathrm{C}$ till it is sunk on the bottom of the container. Transverse frozen sections (15 $\mu \mathrm{m}$ thick) were cut on CM1900 freezing microtome (Leica, Germany). Sections were blocked with $10 \%$ goat serum in phosphate buffered saline (PBS) for $2 \mathrm{~h}$ at room temperature and incubated with primary antibody at $4{ }^{\circ} \mathrm{C}$ overnight. The primary antibodies used are listed in Table 1. After three washes with PBS, the sections were incubated with secondary antibodies for $2-3 \mathrm{~h}$ at room temperature. For double immunofluorescence, sections were incubated with a mixture of primary antibodies overnight at $4{ }^{\circ} \mathrm{C}$, followed by a mixture of FITC-conjugated and Cy3-conjugated secondary antibodies. For IB4 labeling, the Alexafluor 594-conjugated isolectin IB4 from Griffonia simplicifolia was used for bioaffinity labeling. The images were examined under a laser scan confocal fluorescent microscope (Olympus FV1000, Japan).

\section{Western blotting}

Rats were sacrificed by decapitation after behavioral testing, and the L4-6 DRGs ipsilateral to BV injection were obtained and homogenized in a RIPA lysis buffer containing protease inhibitors (Applygen Technologies Inc., China). Protein concentrations of the lysate were determined using a BCA Protein Assay kit (Thermo Scientific, Rockford, IL, USA). Protein samples were heated for $10 \mathrm{~min}$ at $95{ }^{\circ} \mathrm{C}$ with SDS-PAGE sample buffer, and equal amounts of protein were then separated by $10 \%$ separation gels. The resolved proteins were subsequently transferred to nitrocellulose membranes (Bio-Rad, Hercules, CA, USA) followed by the incubation with $5 \%$ nonfat milk (Bio-rad, CA, USA) in PBS with $0.05 \%$ Tween 20 (PBST) for at least $30 \mathrm{~min}$ at room

Table 1 Antibodies for immunofluorescence and Western blotting

\begin{tabular}{lll}
\hline Antibodies & Host & Vendor \\
\hline SDF-1 & Goat & Santa Cruz \\
CXCR4 & Goat & Santa Cruz \\
IB4 & - & Life Technologies \\
Substance P & Rabbit & Chemicon \\
TRPV1 & Rabbit & Alomone \\
PERK & Mouse & Santa Cruz \\
Nav1.8 & Rabbit & Alomone \\
GFAP & Mouse & Millipore \\
\hline
\end{tabular}

temperature. Then, the membranes were incubated with primary antibodies at $4{ }^{\circ} \mathrm{C}$ overnight. The primary antibodies used are listed in Table 1. After washing three times in PBST, the membranes were incubated for $2 \mathrm{~h}$ at room temperature with an HRP-conjugated secondary antibody (1:2000, Bio-Rad). The membranes were visualized with enhanced chemiluminescencce solution (Alpha Innotech Corp.), and the signals were captured with FluorChem FC2 (Alpha Innotech Corp.). The density of specific bands was measured with a computer-assisted imaging analysis system (Bio-Rad, CA, USA) and normalized to $\beta$-tubulin intensity.

\section{Intact DRG preparation and electrophysiological recording}

Rats were anesthetized with $1 \%$ pentobarbital sodium (50 mg/kg, i.p.); L4-6 DRGs were harvested for electrophysiological recording $2 \mathrm{~h}$ after BV injection when the maximal effect was seen for pain hypersensitivity. The whole DRGs were placed into an artificial cerebrospinal fluid maintained at $4{ }^{\circ} \mathrm{C}$ (ACSF, contained in mM: $124 \mathrm{NaCl}, 2.5 \mathrm{KCl}, 1.2 \mathrm{NaH}_{2} \mathrm{PO}_{4}, 1.0 \mathrm{MgCl}_{2}, 2.0 \mathrm{CaCl}_{2}$, $25 \mathrm{NaHCO}_{3}$, and 10 glucose). Then, the DRGs were incubated with digestive solution containing $0.4 \mathrm{mg} / \mathrm{ml}$ trypsin (Sigma) and $1.0 \mathrm{mg} / \mathrm{ml}$ type-A collagenase (Sigma) for $40 \mathrm{~min}$ at $37{ }^{\circ} \mathrm{C}$. After digestion, all DRGs were incubated in carbogen gas-bubbled ACSF at room temperature $\left(25-28{ }^{\circ} \mathrm{C}\right)$ for at least $2 \mathrm{~h}$.

For electrophysiological recording, the ganglion was transferred to the recording chamber which was perfused with carbogen gas-bubbled ACSF at room temperature. A small mesh anchor was used to keep the ganglion stabilized. The neurons in DRG were visualized with $\times 40$ water-immersion objective attached to a BX51WI microscope (BX51WI, Olympus, Japan) equipped with infrareddifferential interference contrast optics. All recordings were made with EPC10 amplifier and Pulse software (HEKA Elektronik, Germany). Current-clamp recordings were made to evoke action potentials and measure the changes of membrane potential using the whole-cell patch-clamp technique. The patch pipettes were fabricated with P-97 Puller (Narishige, Japan) and had resistances of 4-7 M $\Omega$ with internal solution before seal formation. The internal pipette solution contained the following (in mM): $140 \mathrm{KCl}, 2 \mathrm{MgCl}_{2}, 10 \mathrm{HEPES}$, and $2 \mathrm{Mg}$ ATP (pH 7.4, adjusted by $\mathrm{KOH}$ ). Osmolarity was adjusted to 290-300 mOsm by sucrose. All junction potentials were corrected online by adjusting the pipette offset. After G $\Omega$-seal whole-cell formed under voltage-clamp holding at-60 $\mathrm{mV}$, capacitance transient was canceled and serious resistance was compensated (80-90\%) digitally. Neurons were selected for further study if they had a resting membrane potential more negative than $-50 \mathrm{mV}$ and exhibited an overshooting action potential. 


\section{Statistical analysis}

All data were expressed as mean \pm SEM. Differences in changes of values of each group were tested using $t$ tests and one-way ANOVA, followed by individual post hoc comparisons (Tukey or Bonferroni test). A level of $P<0.05$ was accepted as significant.

\section{Results}

Localization of CXCR4 within primary nociceptor neurons Under naïve state, CXCR4 was localized in almost all non-peptidergic IB4-positive cells (Fig. 1a-a2); however, CXCR4 was only seen in a few number of peptidergic SP-positive cells (Fig. 1b-b2). Moreover, CXCR4 was also localized in most of TRPV1-positive neurons (Fig. 1c-c2). CXCR4 was also seen in NF-200 positive neurons (data not shown).
Up-regulation of SDF1 and CXCR4 in the dorsal root ganglia induced by peripheral inflammatory pain state Under peripheral inflammatory pain state induced by i.pl. BV injection, the SDF1-like immunoreactivity was significantly increased in the DRG when compared with the saline control in which less SDF1 immunoreactivity could be seen (Fig. 2a, b). Because the BV-induced increase in SDF1-like immunoreactivity was not seen in the DRG neuronal profiles, but was seen in intercellular space, we performed a double immunofluorescent labeling for SDF1 and glial fibrillary acidic protein (GFAP), a marker for satellite glial cells of the DRG. As seen in Fig. 2c, SDF1 was localized in almost all GFAP-positive profiles in the DRG (Fig. 2c).

We next sought to examine the level of CXCR4 expression in the DRG under BV injection-induced inflammatory pain state. As shown in Fig. $2 \mathrm{~d}-\mathrm{f}$, the expression
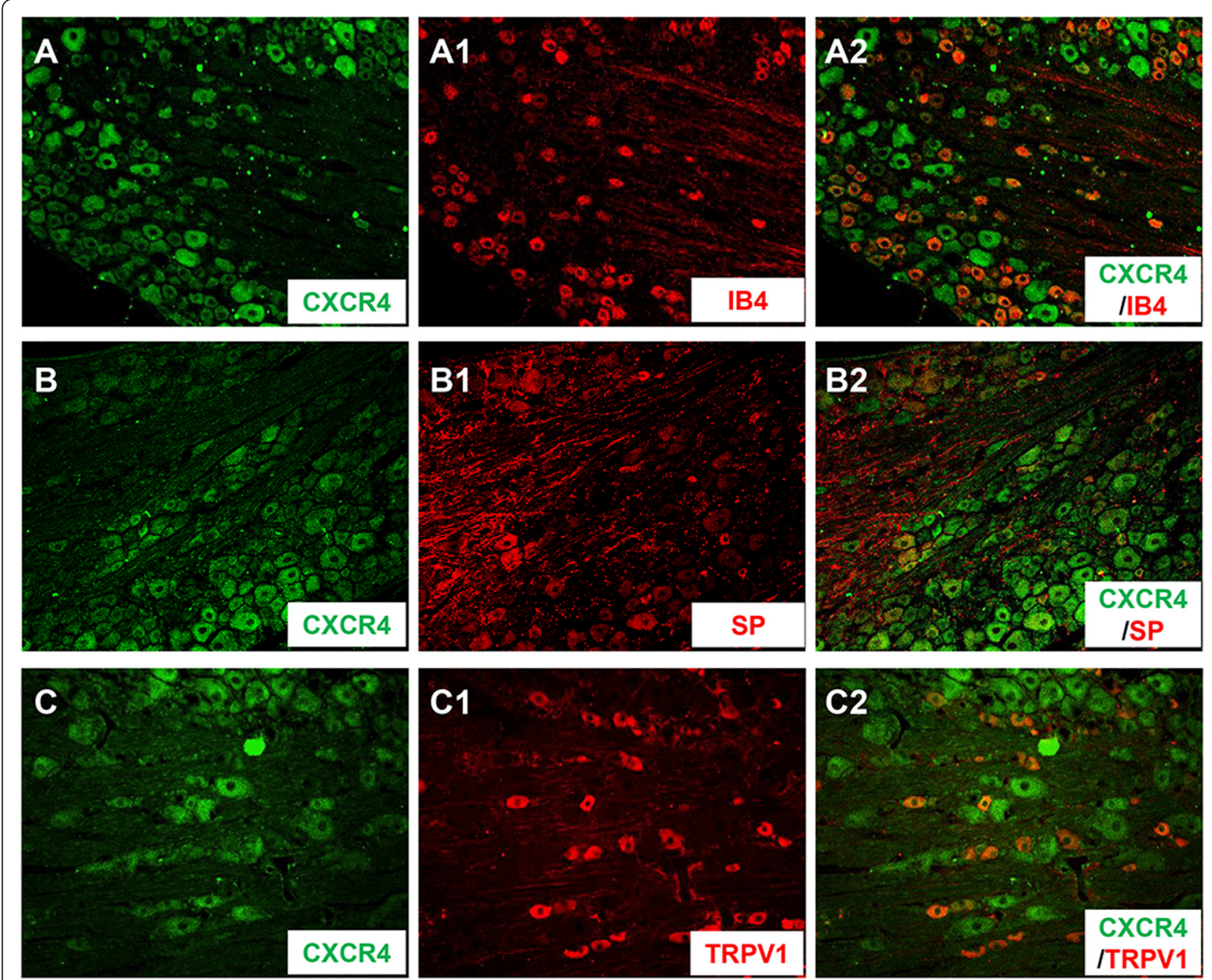

Fig. 1 Localization of CXCR4 in rat primary nociceptive neurons. Immunofluorescence micrographs show the double-staining of CXCR4 (a-c) with IB4 (a1), substance P (SP, b1), and TRPV1 (c1). Showing merged images from a1 and a2, b1 and b2, c1 and $\mathbf{c 2}$. Note that CXCR4 was co-localized with IB4, SP, and TRPV1 in subpopulations of DRG neurons (a2-c2) 


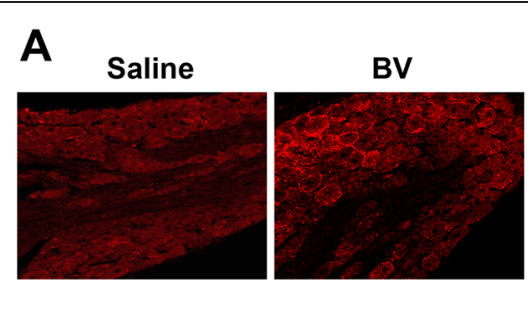

B
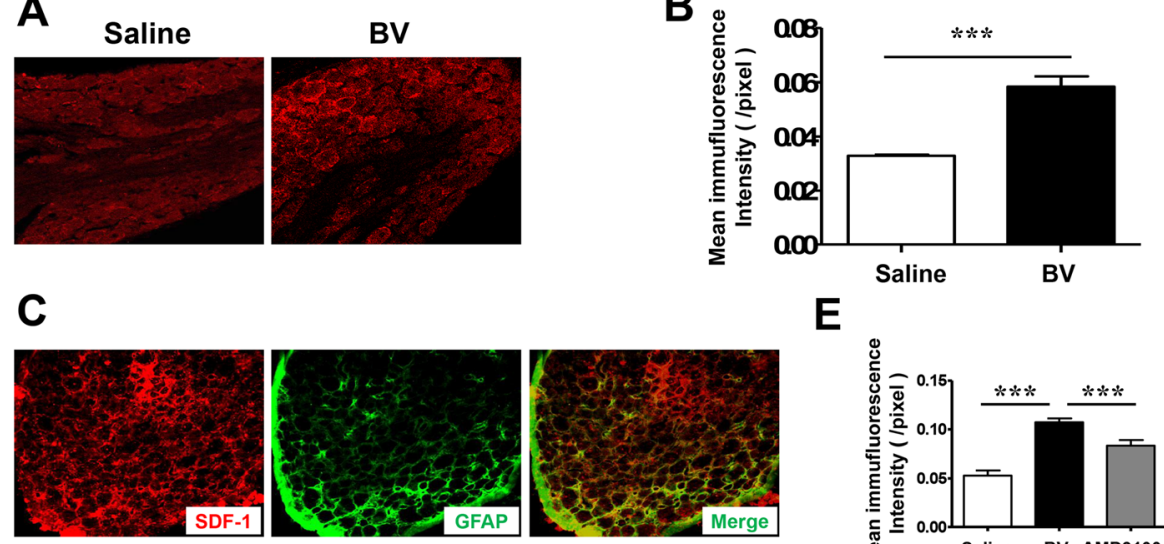

E

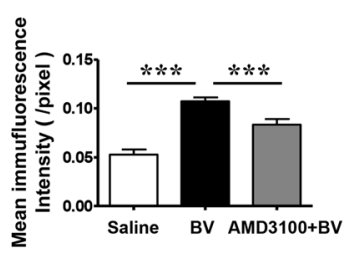

D Saline

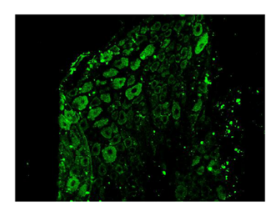

BV

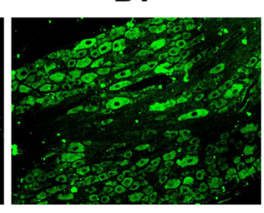

AMD3100+BV

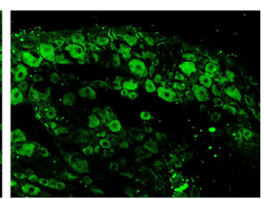

$\mathbf{F}$

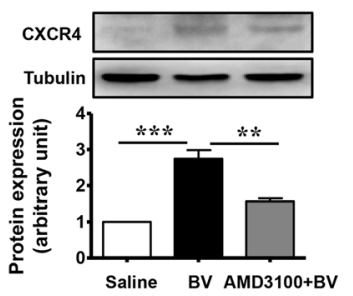

Fig. 2 Intraplantar BV injection induces up-regulation of SDF1 and CXCR4 in the rat lumbar DRG. a Representative immunofluorescence photomicrographs of SDF1 in lumbar DRG from saline-treated rats and BV-treated rats. b Quantification of the mean immunofluorescent intensity of SDF1 showing an increase in SDF1 expression following intraplantar BV injection $\left(n=5 /\right.$ group, $\left.{ }^{* * *} P<0.001\right)$. c Immunofluorescent photomicrographs of double-staining of the SDF1 (red) with GFAP (green), a marker for satellite glial cell. $\mathbf{d}$ Representative immunofluorescent photomicrographs of CXCR4 in the lumbar DRG from saline-treated rats, BV-treated rats, and rats that received AMD3100 10 min before BV injection. e Quantification of the mean immunofluorescent intensity of CXCR4 showing an increase in CXCR4 expression following intraplantar $B V$ injection and a reduction of CXCR4 in rats that received AMD3100 10 min before injection $\left(n=5 /\right.$ group, $\left.{ }^{* * *} P<0.001\right)$. f Western blot showing the expression of CXCR4 in lumbar DRG from saline-treated rats, BV-treated rats, and rats that received AMD3100 10 min before BV injection. Representative bands are shown on the top, and data summary is shown on the bottom. $\left(n=3 /\right.$ group, ${ }^{* *} P<0.01$, $\left.{ }^{* *} P<0.001\right)$

level of CXCR4 was also significantly increased in the DRG neurons relative to saline control demonstrated by both immunofluorescent staining and Western blotting analysis. However, the up-regulation of CXCR4 induced by i.pl. BV injection could be significantly prevented by pre-treatment with subcutaneous AMD3100, a selective CXCR4 inhibitor, suggesting that the BV-induced overexpression of CXCR4 in the DRG cells may be partially mediated by peripheral SDF1-CXCR4 signaling in the skin through trafficking to the peripheral terminals. The BV-induced increases in both SDF1 and CXCR4 were restricted to the ipsilateral side to the BV injection, but with the level of them being unchanged in the contralateral side (not shown).

Inhibition of the BV-induced hyperexcitable state of primary nociceptor neurons by AMD3100, a selective antagonist of CXCR4

Similar to our previous reports $[28,29,40,41]$, the primary nociceptor neurons could be persistently activated by peripheral inflammatory pain state induced by i.pl. injection of both bee venom and complete Freund's adjuvant (CFA) through up-regulation of both Nav1.8 and Nav1.9 of VGSC in the DRG cells. However, only the tonic, but not the phasic, type of primary nociceptor neurons was involved in this processing [41]. The phasic type of the DRG neurons is characterized by producing only one AP following repeated electrical stimulation; however, the tonic type is featured by producing many APs following the same electrical stimulation [41].

Figure 3a showed an example of current patch-clamp recording of tonic type of primary nociceptor neurons in which the firing rate was significantly increased in the BV-treated group relative to the saline control (left panel of Fig. 3a). The averaged number of APs produced in the BV-treated group was about fivefold more than the saline control group (Fig. 3e, ${ }^{* *} P<0.01 \mathrm{BV}$ vs. saline, $n=10$ cells for each group). In the BV-treated group, the rheobase and half-width of APs were significantly reduced relative to saline control group (Fig. 3c, d ${ }^{*} P<0.05 \mathrm{BV}$ vs. saline, $n=10$ cells for each group); 
A

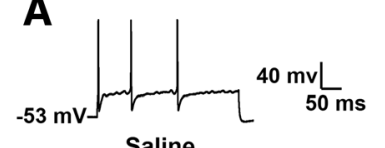

Saline

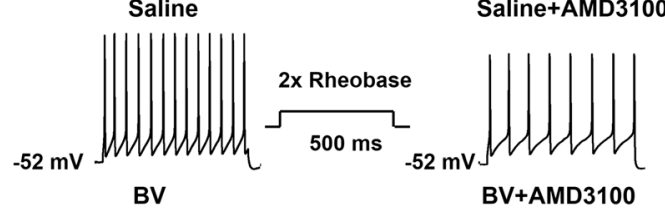

C

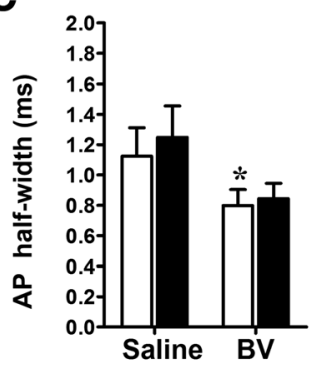

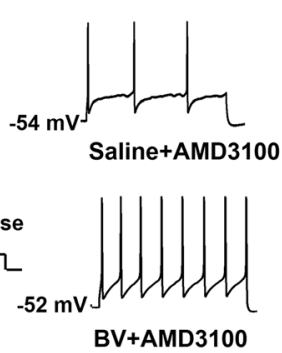

D

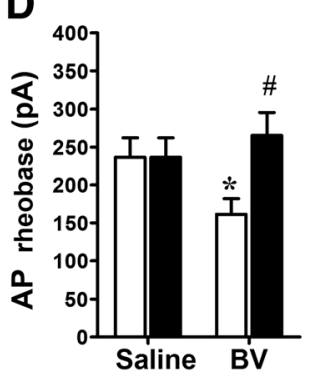

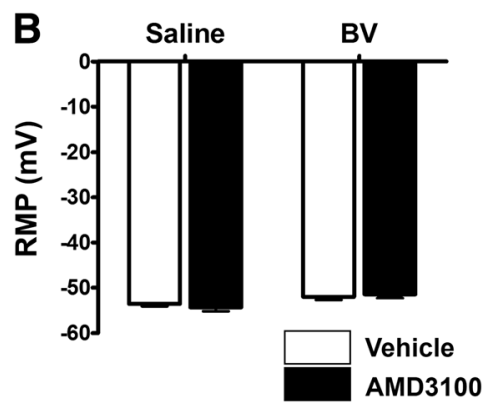

E

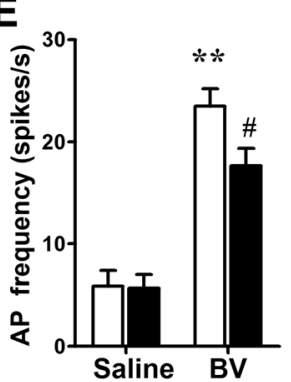

Fig. 3 Reversal of BV-induced hyperexcitability of small- and medium-sized DRG neurons by antagonism of CXCR4. a Representative traces of current-evoked action potentials (AP) in the DRG neurons harvested from saline-treated and BV-treated rats. b-e Histograms exhibiting the effect of bath application of AMD3100, a selective antagonist of CXCR4, on the absolute values of RMP (b), AP half-width (c), AP rheobase (d), and AP frequency (e) in DRG neurons from saline-treated and BV-treated rats. $R M P$ rest membrane potential, $A P$ action potential; $n=8 /$ group, $P<0.05$, ${ }^{* *} P<0.01 \mathrm{BV}+$ vehicle vs. saline + vehicle; $\# P<0.05$ BV + AMD3100 vs. saline + AMD3100

however, the resting membrane potential (RMP) was not changed between the two groups of DRG neurons (Fig. 3b). These results were also consistent with our previous report [41].

To test the role of CXCR4 in mediation of the changed excitability of DRG neurons, the effects of bath perfusion with AMD3100 $(1 \mu \mathrm{M})$ to the DRG neurons were studied. Bath perfusion of AMD3100 could inhibit the firing rate $(\# P<0.05$ AMD3100 vs. vehicle, $n=10$ for each group) and reverse the lowered rheobase value to the normal level $(\# P<0.05$ AMD3100 vs. vehicle, $n=10$ for each group); however, the drug had no effect on the lowered AP half-width in the BV-treated group (Fig. 3). Bath perfusion of AMD3100 had no effect on either membrane properties or firing rate in saline control group (Fig. 3).

\section{Suppression of BV-induced up-regulation of Nav1.8 and phosphorylated ERK by blocking CXCR4}

Based upon our present and previous data, it is reasonable to link the functions of SDF1-CXCR4 signaling with expression of Nav1.8 due to the following accumulating evidence: (1) the hyperexcitability of the tonic type of DRG neurons induced by peripheral inflammatory pain state was associated with up-regulation of Nav1.8 and Nav1.9 $[28,29,41],(2)$ anti-sense down-regulation of Nav1.8 or Nav1.9 resulted in reversal of altered excitability of DRG neurons induced by peripheral inflammatory pain state $[28,29]$, and (3) the hyperexcitability of the tonic type of DRG neurons induced by peripheral inflammatory pain state could be blocked by antagonism against CXCR4 with AMD3100 (see in the current study). Thus, we proposed that activation of SDF1-CXCR4 signaling should be involved in regulation of Nav1.8 expression through an unknown molecular downstream route. Because it has been suggested that ERK, a subfamily of mitogenactivated protein kinase (MAPK), is a common intracellular downstream messenger of SDF1-CXCR4 signaling [42-44], examination of the roles of ERK is also reasonable.

We therefore firstly evaluated whether the BV-induced overexpression of Nav1.8 could be blocked by antagonism against CXCR4 and inhibition of activation of ERK. As shown in Fig. 4a and b, pre-treatment with i.pl. AMD3100 and i.t. U0126, respectively, could remarkably block the increased expression of Nav1.8 in the DRG neurons induced by i.pl. BV injection. Using Western blot, it was also confirmed that the BV-induced upregulation of both Nav1.8 and phosphorylated form of ERK could be blocked by i.pl. AMD3100 as well as by i.t. U0126 (Fig. 4c and d). Moreover, as shown in Fig. 4e, pre-treatment with i.pl. U0126 could also block the increased expression of Nav1.8 in the DRG neurons induced by i.pl. BV injection. 

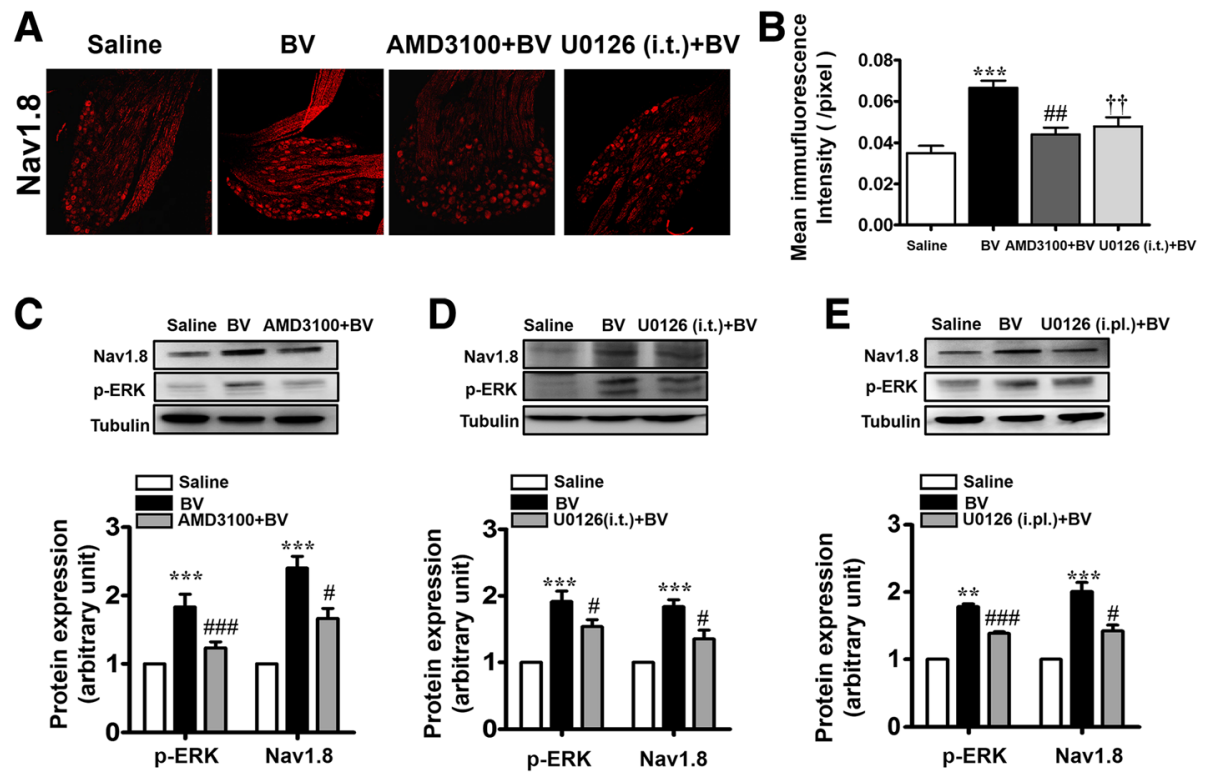

Fig. 4 Blockade of BV-induced phosphorylation of ERK and up-regulation of Nav1.8 protein expression in the lumbar DRG by peripherally local injection of AMD3100. a Representative immunofluorescent photomicrographs showing expression of Nav1.8 in the lumbar DRG from saline-treated rats, BV-treated rats, and rats receiving intraplantar AMD3100 or intrathecal U0126 10 min before intraplantar BV injection. b Quantification of the mean immufluorescent intensity of Nav1.8 showing an increase in expression following intraplantar (i.pl.) BV injection, and a reduction in rats receiving AMD3100 or U0126 administration 10 min prior to BV $\left(n=5 /\right.$ group, ${ }^{* * *} P<0.001$, \#\#P<0.001, $++P<0.001$ vs. saline). c Western blot showing that AMD3100 prevented the BV-induced up-regulation of Nav1.8 and pERK activation in lumbar DRG from occurring ( $n=3 /$ group, ${ }^{* * *} P<0.001$ vs. saline; \#P<0.05, \#\#\#P<0.001 vs. BV). $\mathbf{d}$ Western blot showing that intrathecal U0126 prevented the BV-induced up-regulation of Nav1.8 and pERK activation in lumbar DRG from occurring ( $n=3 /$ group, ${ }^{* *} P<0.001$ vs. saline; $\# P<0.05$ vs. BV). e Western blot showing that intraplantar U0126 prevented the BV-induced up-regulation of Nav1.8 and pERK activation in lumbar DRG from occurring $\left(n=3\right.$ /group, ${ }^{* *} P<0.01$, ${ }^{* *} P<0.001$ vs. saline; $\# P<0.05, \# \# \#<0.001$ vs. BV)

Relief of BV-induced persistent spontaneous nociception and primary pain hypersensitivity by peripherally local injection of AMD3100

During the 1-h time course of the BV-induced PSN, peripherally local pre-treatment with AMD3100 (100 and $200 \mu \mathrm{g} / 20 \mu \mathrm{l})$ resulted in a dose-related suppressive effect on the development of paw-flinching reflex relative to the vehicle control, while the lowest dose $(10 \mu \mathrm{g} /$ $20 \mu \mathrm{l}$ ) had no significant effect (Fig. 5a). The averaged inhibition rate produced by AMD3100 was $26.90 \%$ for $100 \mu \mathrm{g}$ and $40.17 \%$ for $200 \mu \mathrm{g}$, respectively (Fig. 5b).

As for the effects of AMD3100 on the pain hypersensitivity, only the highest dose of AMD3100 $(200 \mu \mathrm{g} / 20 \mu \mathrm{l})$ could significantly prevent the development of both primary heat and mechanical hypersensitivity but with the mirror-image heat hypersensitivity being unaffected (Fig. 5c, d). The same treatment of AMD3100 even at the highest dose used in the present study had no influence upon the basal pain sensitivity to either heat or mechanical stimuli (data not shown).

Involvement of ERK signaling and Nav1.8 in the development and maintenance of BV-induced persistent spontaneous nociception and primary pain hypersensitivity As shown in Figs. 6a and 7a, i.pl. BV injection produced a rapid-onset, long-term spontaneous flinching reflex which could last for 1-2 h. Comparing with vehicletreated control group, i.t. pre-treatment with U0126 $(10 \mu \mathrm{g} / 10 \mu \mathrm{l}, 10 \mathrm{~min}$ before) significantly prevented the development of persistent spontaneous nociception (Fig. 6a, b). Likewise, ipsilateral i.pl. treatment with A$803467(500 \mu \mathrm{g} / 50 \mu \mathrm{l}) 10 \mathrm{~min}$ before BV injection also prevented the occurrence of persistent flinching reflex (Fig. 7a, b).

In comparison with vehicle group, pre-treatment with i.t. U0126 $(10 \mu \mathrm{g} / 10 \mu \mathrm{l})$ prevented the development of primary mechanical and heat hypersensitivity identified in the hindpaws ipsilateral to BV injection and the mirror-image heat hypersensitivity in the contralateral hindpaws (Fig. 6c, d). Figure 7c and $d$ showed that the established primary mechanical and heat hypersensitivity could be reversed by i.pl. treatment with A-803467 $(500 \mu \mathrm{g} / 50 \mu \mathrm{l}) 90 \mathrm{~min}$ post-BV injection, while the mirror-image heat hypersensitivity remained unaltered.

\section{Discussion}

In the present study, we demonstrated that the SDF1CXCR4 signaling contributes to the hyperexcitability of tonic type of the primary nociceptor cells through upregulation of Nav1.8 via regulating ERK pathway. Blocking the SDF1-CXCR4 signaling as well as ERK activation and Nav1.8 activity can suppress both the persistent 

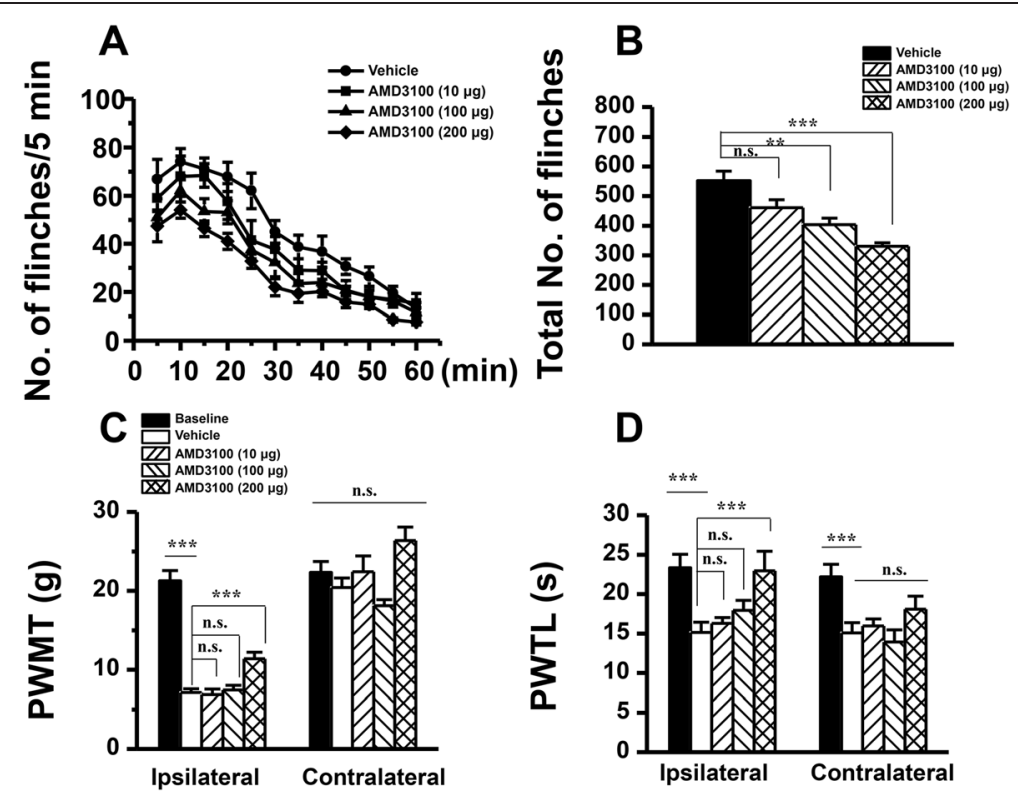

Fig. 5 Effects of peripherally local injection of AMD3100 on BV-induced persistent spontaneous nociception and mechanical and thermal hyperalgesia. a Curve graphs showing the effect of AMD3100 on the persistent spontaneous nociception evaluated by recording the spontaneous paw flinches at each $5 \mathrm{~min}$ interval for $1 \mathrm{~h}$ immediately after intraplantar BV injection. b Column graphs showing the effect of AMD3100 on the mean total numbers of paw flinches averaged from the $1 \mathrm{~h}$ period intraplantar BV injection. $\mathbf{c}$, d Exhibiting the effects of AMD3100 on the PWMT and PWTL measured in bilateral hindpaws of BV-treated rats. PWMT paw withdrawal mechanical threshold, PWTL paw withdrawal thermal latency; ${ }^{* *} P<0.01,{ }^{* * *} P<0.001$, $n=8-10$ for each group
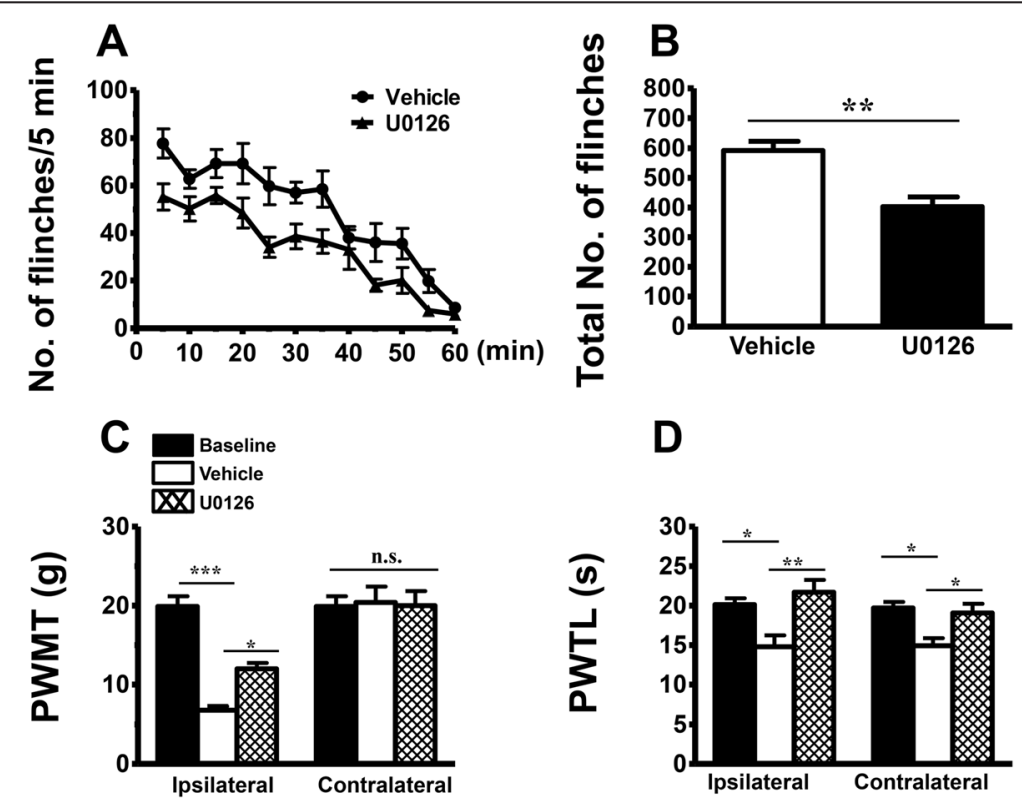

Fig. 6 Effects of intrathecal administration of an ERK inhibitor, U0126, on BV-induced persistent spontaneous nociception and mechanical and thermal hyperalgesia. a Curve graphs showing the effect of $U 0126$ on the persistent spontaneous nociception evaluated by recording the spontaneous paw flinches at each 5 min interval for $1 \mathrm{~h}$ immediately after intraplantar BV injection. b Column graphs showing the effect of U0126 on the mean total numbers of paw flinches averaged from the $1 \mathrm{~h}$ period intraplantar BV injection. c, $\mathbf{d}$ Exhibiting the effects of U0126 on the PWMT and PWTL measured in bilateral hindpaws of BV-treated rats. PWMT paw withdrawal mechanical threshold, $P W T L$ paw withdrawal thermal latency; ${ }^{*} P<0.05,{ }^{* *} P<0.01$, ${ }^{* * *} P<0.001, n=6$ for each group 

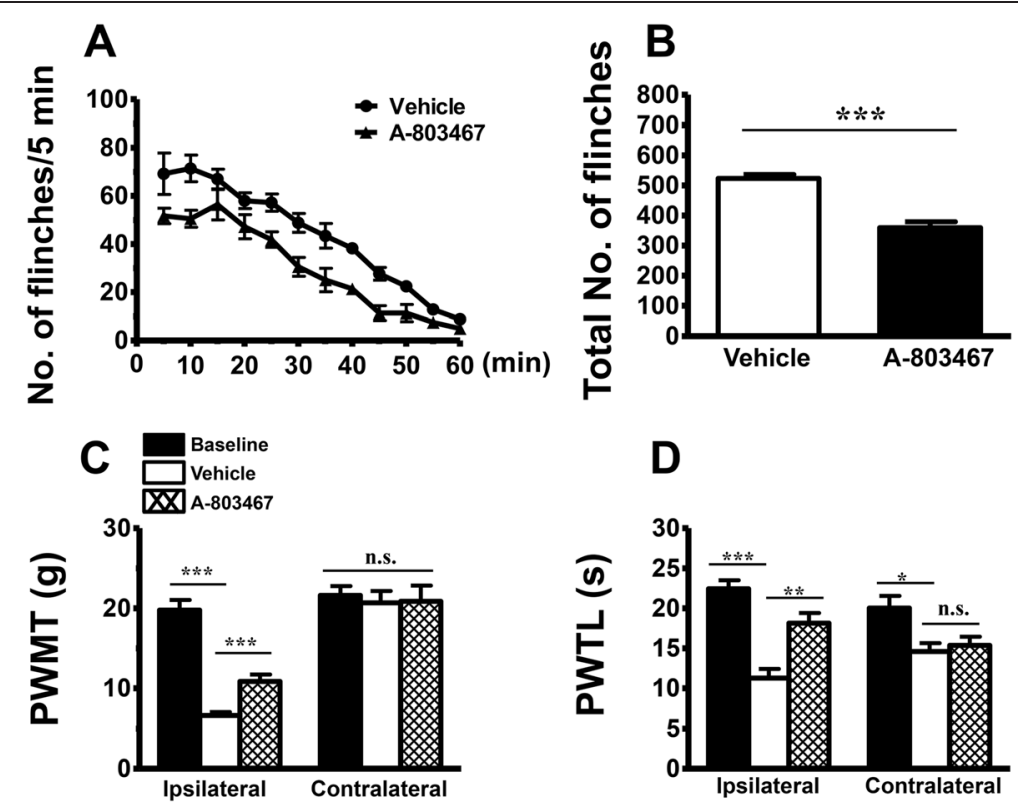

Fig. 7 Effects of intraplanter injection of a Nav1.8 blocker, A-803467, on BV-induced persistent spontaneous nociception and mechanical and thermal hyperialgesia. a Curve graphs showing the effect of A-803467 on the persistent spontaneous nociception evaluated by recording the spontaneous paw flinches at each 5 min interval for $1 \mathrm{~h}$ immediately after intraplantar BV injection. b Column graphs showing the effect of A-803467 on the mean total numbers of paw flinches averaged from the $1 \mathrm{~h}$ period intraplantar BV injection. c, d Exhibiting the effects of A-803467 on the PWMT and PWTL measured in bilateral hindpaws of BV-treated rats. PWMT paw withdrawal mechanical threshold, $P W T L$ paw withdrawal thermal latency; ${ }^{*} P<0.05$, ${ }^{* *} P<0.01,{ }^{* * *} P<0.001, n=6$ for each group

spontaneous nociception and primary pain hypersensitivity through down-regulation of both ERK and Nav1.8. Taken together, it is concluded that SDF1-CXCR4 signaling contributes to persistent pain and hyperalgesia (and allodynia) via regulating the excitability of primary nociceptive neurons maintained by ERK-dependent Nav1.8 up-regulation.

\section{Maintenance of primary nociceptor neuronal hyperexcitability by SDF1-CXCR4 signaling under peripheral inflammatory pain state}

In the present study, it is interesting to note that antagonism of CXCR4 by bath perfusion of DRG with AMD3100 results in significant inhibition of the BVenhanced neuronal firing rate by reversing the lowered rheobase value to the normal level. This result suggests a maintaining role of SDF1-CXCR4 signaling in the primary nociceptor hyperexcitability at the neuronal cell body level caused by peripheral inflammatory pain state. The primary nociceptor neuronal cell body hyperexcitability caused by the BV-induced peripheral inflammatory pain state has already been demonstrated in our previous reports [28, 29, 40, 41]. Regarding this, one question may arise to be asked: which cell types are possible sources of SDF1 and CXCR4 in the DRG? To answer this question, we further demonstrated that, by double immunofluorescent staining and/or Western blotting, SDF1 was exclusively up-regulated in GFAP- positive non-neuronal SGCs of the DRG following i.pl. BV injection, while CXCR4 was mainly co-localized with IB4, SP, and TRPV1, specific biomarkers of primary nociceptor neurons in the DRG. This at least suggests that the non-neuronal SGCs can be activated by peripheral inflammatory pain state that serve as a source of SDF1 release, allowing its binding to CXCR4 be over expressed in the primary nociceptor neuronal cell body caused by persistent firing initiated in the peripheral terminals [33, 45-47]. The mechanism of the activation of non-neuronal SGCs may be due to the fractalkines released from DRG neurons which has been demonstrated by Souza and colleagues in i.pl. carrageenan injectioninduced inflammatory pain model [48]. It has been demonstrated that, under physiological state, both primary afferent neurons and non-neuronal SGCs in the DRG constitutively contain SDF1 that is maintained at a very low level [9]. However, the source of SDF1 under pathological level may vary depending upon different conditions. In antiretroviral toxic neuropathy model, Bhangoo and colleagues have observed up-regulation of SDF1 and CXCR4 mRNA at 7 and 14 days after administration of antiretroviral drug 2, 3-dideoxycytidine, and the enhanced expression of SDF1 was mostly observed in the DRG non-neuronal cells [49]. Similarly, Dubovy and colleagues have also demonstrated that the non-neuronal SGCs were the source of SDF1 by showing co-localization of SDF1 and glutamine synthetase in the DRG of animals 
with neuropathic pain induced by CCI [15]. Based upon the above lines of evidence, it is highly suggested that the inducible release of SDF1 from non-neuronal SGCs by peripheral inflammatory and neuropathic pain conditions should result in development of intraganglionar inflammatory microenvironment, by which long-term hyperexcitability of primary nociceptor neurons can be maintained. As lines of supporting evidence, it has been demonstrated that the SGCs in the DRG when they had been activated by i.pl. carrageenan injection could produce some proinflammatory mediators (TNF $\alpha$, IL-1 $\beta$, and prostanoids) that can directly excite the primary nociceptive neurons [48]. Moreover, SDF1 has been shown to significantly increase intracellular calcium concentrations in the isolated DRG neurons under several persistent pain conditions [49-51]. It has also been demonstrated that exogenous SDF1 could lower the threshold for action potential generation and depolarize nociceptive DRG neurons in cultured DRG neurons [21]. However, sensory neurons of the DRG may also be the source of SDF1 since increase in SDF1 mRNA or protein have been observed in DRG neurons of transgenic mice with high-fat diet-induced type II diabetic neuropathy [50] and animals receiving repeated morphine treatment [51]. Besides SDF1, other chemokines are also likely to be involved in primary sensory neuronal hyperexcitability, because up-regulation of CCL2, CCL3, and CXCL1 by pain and direct activation of DRG neurons by them have been seen in several previous reports $[6,30,31,52,53]$.

As for the source of CXCR4, a selective cognate receptor of SDF1, only primary sensory neurons have been targeted. Consistent with some previous reports $[9,16,21]$, in our present study, CXCR4 was constitutively present but overexpressed under peripheral inflammatory pain state in both non-peptidergic (IB4-positive) and peptidergic (SP-positive) primary nociceptor neurons. It is also co-localized with TRPV1, a thermonociceptor of primary sensory afferent. However, the overexpression of CXCR4 induced by intraplantar BV injection could be significantly prevented by pre-treatment with i.pl. AMD3100, a selective CXCR4 inhibitor, suggesting that the BV-induced overexpression of CXCR4 in the DRG cells may be partially mediated by peripheral SDF1-CXCR4 signaling in the skin which was trafficking from neuronal soma to the peripheral terminals. In the case of SDF1-CXCR4 signaling pathway, Reaux-Le and colleagues have found that CXCR4 receptor was constitutively present in both peptidergic (CGRP positive) and non-peptidergic (IB4 positive) DRG neuronal soma in rats [9]. They have also demonstrated that CXCR4 can be localized in pre-synaptic components of both type I and type II glomeruli in the spinal dorsa horn under electron microscope, indicating that CXCR4 could be axonally transported to both peripheral and central terminals of the primary afferent neurons in DRG and exert its functions
[9]. Although we do not detect the protein level of CXCR4 in the skin directly in the current study, Reaux-Le and colleagues' results can lend support to the rationale of peripheral administration of AMD3100 in our current study because CXCR4 immunoreactivities co-expressed in the CGRP-positive fibers have been shown to be present in the glabrous skin as well as in the DRG cells and project to the dermis [9]. The functional nature of this increased CXCR4 receptor expression was identified by our behavioral pharmacology assays in which pre-treatment with i.pl. AMD3100 significantly prevented the development of the BV-induced persistent spontaneous pain-related behaviors and pain hypersensitivity. Moreover, bath perfusion of medium- and small-sized DRG neurons with AMD3100 also significantly reduced BV-induced tonic discharges by restoration of rheobase value to normal level, suggesting a maintaining role of SDF1-CXCR4 signaling in the primary nociceptor hyperexcitability. Since it is known that the decrease in rheobase value may reflect changes in persistent $\mathrm{Na}+$ conductance $[54,55]$, the roles of TTX-resistant VGSC $\alpha$ subunits Nav1.8 and/or Nav1.9, which are selectively expressed in the primary nociceptor neurons, should be further investigated. As aforementioned, we have already demonstrated that Nav1.8 and Nav1.9 could be upregulated in the small and medium-sized DRG cells by i.pl. melittin, the major toxin of whole bee venom, or CFA injection, resulting in increased persistent current mediated by Nav1.8 and Nav1.9 and enhanced firing rate of tonic, but not phasic, type of primary nociceptor neurons with lowered rheobase value $[28,29,41]$. Down-regulation of Nav1.8 and Nav1.9 by anti-sense oligodeoxynucleotide, respectively, resulted in restoration of Nav1.8 and Nav1.9 current density and rheobase value, leading to reduction of hyperexcitability of primary nociceptor neurons and relief of persistent nociception and pain hypersensitivity induced by i.pl. injection of melittin or CFA [28, 29]. In the present, our behavioral data also demonstrated that peripheral pretreatment with A-803467, a selective Nav1.8 blocker, could inhibit the persistent nociception and reverse the primary pain hypersensitivity induced by i.pl. BV injection, indicating that increased expression of Nav1.8 in the DRG induced by i.pl. BV injection contributed to the development and maintenance of the BV-induced pain-related behaviors. Taken together, we proposed that there must be a functional link between SDF1-CXCR4 signaling and expression of Nav1.8 or Nav1.9 that maintains primary nociceptor neuronal hyperexcitability under peripheral inflammatory pain state.

\section{Involvement of SDF1-CXCR4 signaling in the up-regulation of Nav1.8 sodium channels in DRG neurons via ERK-dependent pathway}

In the present study, we further demonstrated that peripheral antagonism against CXCR4 pharmacologically 
with AMD3100 10 min prior to i.pl. BV could prevent up-regulation of Nav1.8 sodium channel from occurrence in the DRG. The phosphorylation of ERK (pERK), an isoform of MAPK, was also significantly suppressed in the DRG by pre-treatment of AMD3100 at the i.pl. BV injection site. The phosphorylation of ERK has been shown to be critical for up-regulation of Nav1.8 [56]. CXCR4 activation has also been shown to induce multiple intracellular signal transduction pathways, including ERK signaling pathway [42-44]. Both intrathecal and intraplantar pre-treatment of U0126, an ERK inhibitor, could inhibit the level of pERK and expression of Nav1.8 in the DRG. Furthermore, consistent with our previous findings that peripheral ERK contributes to the persistent pain induced by melittin, we here found that the BV-induced persistent nociception and primary mechanical and thermal hypersensitivity could be inhibited by pre-treatment with i.t. U0126 and i.pl. A-803467, respectively. These results suggest that SDF1-CXCR4 signaling should be involved in up-regulation of Nav1.8 through an ERK-dependent pathway in the primary nociceptor neurons following i.pl. BV injection, which contributes to hyperexcitability of the primary nociceptor neurons that mediate maintenance of both persistent spontaneous nociception and primary pain hypersensitivity. In support, up-regulation of Nav1.8 sodium channel by chemokine CCL2 has been seen in acutely dissociated and cultured DRG neurons from naïve rats that are likely to be mediated by $P K C-\mathrm{NF} K \mathrm{~B}$ and $\mathrm{G} \beta \mathrm{\gamma}$ dependent mechanisms $[30,57,58]$. The expression level of Nav1.8 has also been demonstrated to be upregulated by TNF $\alpha$ or down-regulated by interleukin-10 (IL-10) in rat DRG neurons [26, 59]. However, unlike the release of SDF1 from the SGCs, the source of chemokine CCL2 and pro-inflammatory mediators TNF $\alpha$ and IL-10 were thought to be released through paracrine or autocrine from DRG neurons that possess their respective receptors [26, 30, 57-59]. Taken ours and previous results together, it seems that there are at least two regulating patterns to be involved in up-regulation of Nav1.8 in the DRG under peripheral inflammatory and neuropathic pain conditions: one is likely to be initiated by neuronal activity-dependent autocrine-autoreceptor pattern mediated by TNF $\alpha$-TNFR and/or CCL2-CCR2 signaling pathways within the primary nociceptor neurons $[26,30,57-59]$, while the other is likely to be initiated by SGC-neuronal pattern mediated by SDF1CXCR4 signaling pathway. We propose that the autocrine-autoreceptor pattern should be involved in the early induction process, while the satellite glial-neuronal pattern in the late maintaining process of both peripheral inflammatory and neuropathic pain conditions. As a line of supporting evidence, pro-inflammatory mediator TNF $\alpha$ has been shown to be only involved in the induction of primary nociceptor hyperexcitability but not involved in the maintaining process $[26,59]$. Contrarily, the SGCneuronal pattern mediated by SDF1-CXCR4 signaling pathway was shown, in the current study, to be involved in the maintaining process because the well-established BV-induced primary nociceptor neuronal firing could be suppressed by bath perfusion of the DRG with AMD3100, thus providing with a novel molecular target for treatment of clinical pain.

Nav1.8 is a sensory neuron-specific channel which acts as a major contributor to the upstroke of action potentials and supports repetitive firing in response to depolarizing input, and is preferentially expressed in nociceptive DRG and trigeminal ganglion neurons [24, 60-62]. By using diphtheria toxin to kill all Nav1.8-positive sensory neurons, mechanical, cold, and inflammatory pain have been demonstrated to be ameliorated [63]. Nav1.8-null mutant mice have been shown to have weak responses to noxious cold and mechanical stimulation [64]. Moreover, intraperitoneal administration of A-803467, a potent Nav1.8 sodium channel blocker, has been shown to produce significant anti-nociception in inflammatory pain models induced by CFA and capsaicin [65]. Collectively, these findings suggest that Nav1.8 may be a good target for the development of novel analgesics for treatment of inflammatory pain hypersensitivity [66]. However, the side effects, which were produced due to the high degree of structural homology within the VGSC family, and the poor bioavailability of the existing VGSC blockers limit their clinical use [66-68]. Based upon our present result, targeting the SDF1-CXCR4 signaling between SGCneuronal link might be a good option for treatment of chronic pain. Because the SDF1-CXCR4 signaling may also regulate other various downstream ion channels such as other subtypes of VGSC, voltage-gated calcium channels, and the family of TRP (TRPV, TRPA, TRPM, TRPC) channels, further intensive studies of the regulating functions of SDF1-CXCR4 signaling at the DRG neuronal cell body level may shed new light on the treatment of chronic inflammatory and neuropathic pain conditions.

\section{Conclusions}

In summary, our present data demonstrated that, under the peripheral inflammatory pain condition, SDF1-CXCR4 signaling between non-neuronal SGCs and primary nociceptor neurons is dramatically enlarged through up-regulation of both substances in the DRG which acts as a mediator for the intraganglionar neuroinflammatory microenvironment and induces ERK-dependent up-regulation of Nav1.8 that contributes to the maintaining process of primary nociceptor neuronal hyperexcitability that is required for maintenance of persistent spontaneous pain and hypersensitivity. 


\section{Competing interests}

The authors declare that they have no competing interests.

\section{Authors' contributions}

$J C$ and FY conceived and designed the experiments. FY and WS performed the experiments. YW, C-LL, HF, FY and TH assisted with western blotting assay, behavioral test, tissue and drug preparation. FY and JC analyzed and interpreted the data. YY and X-LW supplied SPF animals. JC and FY wrote the paper. All authors read and approved the final manuscript.

\section{Acknowledgements}

This work was supported by the National Natural Science Foundation of China (81171049), the National Basic Research Development Program of China (2011CB504100, 2013CB835100), the National Key Technology R\&D Program (2013BAI04B04), and the Twelfth Five-Year project (AWS12J004) to JC.

\section{Author details}

${ }^{1}$ Institute for Biomedical Sciences of Pain, Tangdu Hospital, The Fourth Military Medical University, \#569 Xinsi Road, Baqiao, Xi'an 710038, People's Republic of China. ${ }^{2}$ Key Laboratory of Brain Stress and Behavior, PLA, Xi'an 710038, People's Republic of China. ${ }^{3}$ Beijing Institute for Brain Disorders, Beijing 100069, People's Republic of China.

\section{Received: 10 July 2015 Accepted: 18 November 2015}

\section{Published online: 24 November 2015}

\section{References}

1. Ma W, Quirion R. Inflammatory mediators modulating the transient receptor potential vanilloid 1 receptor: therapeutic targets to treat inflammatory and neuropathic pain. Expert Opin Ther Targets. 2007;11:307-20.

2. Gosselin RD, Dansereau MA, Pohl M, Kitabgi P, Beaudet N, Sarret $P$, et al. Chemokine network in the nervous system: a new target for pain relief. Curr Med Chem. 2008;15:2866-75.

3. Cao DL, Zhang ZJ, Xie RG, Jiang BC, Ji RR, Gao YJ. Chemokine CXCL1 enhances inflammatory pain and increases NMDA receptor activity and COX-2 expression in spinal cord neurons via activation of CXCR2. Exp Neurol. 2014;261:328-36.

4. Ji RR, Xu ZZ, Gao YJ. Emerging targets in neuroinflammation-driven chronic pain. Nat Rev Drug Discov. 2014;13:533-48.

5. Turner MD, Nedjai B, Hurst T, Pennington DJ. Cytokines and chemokines: at the crossroads of cell signalling and inflammatory disease. Biochim Biophys Acta. 1843;2014:2563-82.

6. Miller RJ, Jung H, Bhangoo SK, White FA. Cytokine and chemokine regulation of sensory neuron function. Handb Exp Pharmacol. 2009;194:417-49.

7. White FA, Wilson NM. Chemokines as pain mediators and modulators. Curr Opin Anaesthesiol. 2008;21:580-5.

8. Reaux-Le GA, Van Steenwinckel J, Rostene W, Melik PS. Current status of chemokines in the adult CNS. Prog Neurobiol. 2013;104:67-92.

9. Reaux-Le GA, Rivat C, Kitabgi P, Pohl M, Melik PS. Cellular and subcellular localization of CXCL12 and CXCR4 in rat nociceptive structures: physiological relevance. Eur J Neurosci. 2012;36:2619-31.

10. Klein RS, Rubin JB, Gibson HD, DeHaan EN, Alvarez-Hernandez X, Segal RA, et al. SDF-1 alpha induces chemotaxis and enhances Sonic hedgehoginduced proliferation of cerebellar granule cells. Development. 2001;128:1971-81.

11. Wang Y, Deng Y, Zhou GQ. SDF-1alpha/CXCR4-mediated migration of systemically transplanted bone marrow stromal cells towards ischemic brain lesion in a rat model. Brain Res. 2008;1195:104-12.

12. Banisadr G, Frederick TJ, Freitag C, Ren D, Jung H, Miller SD, et al. The role of CXCR4 signaling in the migration of transplanted oligodendrocyte progenitors into the cerebral white matter. Neurobiol Dis. 2011;44:19-27.

13. Virgintino D, Errede M, Rizzi M, Girolamo F, Strippoli M, Walchli T, et al. The CXCL12/CXCR4/CXCR7 ligand-receptor system regulates neuro-glio-vascular interactions and vessel growth during human brain development. J Inherit Metab Dis. 2013;36:455-66.

14. Li M, Ransohoff RM. Multiple roles of chemokine CXCL12 in the central nervous system: a migration from immunology to neurobiology. Prog Neurobiol. 2008;84:116-31.
15. Dubovy P, Klusakova I, Svizenska I, Brazda V. Spatio-temporal changes of SDF1 and its CXCR4 receptor in the dorsal root ganglia following unilateral sciatic nerve injury as a model of neuropathic pain. Histochem Cell Biol. 2010;133:323-37.

16. Knerlich-Lukoschus F, von der Ropp-Brenner B, Lucius R, Mehdorn HM, Held-Feindt J. Spatiotemporal CCR1, CCL3(MIP-1alpha), CXCR4, CXCL12(SDF-1alpha) expression patterns in a rat spinal cord injury model of posttraumatic neuropathic pain. J Neurosurg Spine. 2011;14:583-97.

17. Callewaere C, Banisadr G, Desarmenien MG, Mechighel P, Kitabgi P, Rostene $\mathrm{WH}$, et al. The chemokine SDF-1/CXCL12 modulates the firing pattern of vasopressin neurons and counteracts induced vasopressin release through CXCR4. Proc Natl Acad Sci U S A. 2006;103:8221-6.

18. Guyon A, Nahon JL. Multiple actions of the chemokine stromal cell-derived factor-1alpha on neuronal activity. J Mol Endocrinol. 2007;38:365-76.

19. Guyon A, Rovere C, Cervantes A, Allaeys I, Nahon JL. Stromal cell-derived factor-1alpha directly modulates voltage-dependent currents of the action potential in mammalian neuronal cells. J Neurochem. 2005;93:963-73.

20. Guyon A, Skrzydelsi D, Rovere C, Rostene W, Parsadaniantz SM, Nahon JL. Stromal cell-derived factor-1alpha modulation of the excitability of rat substantia nigra dopaminergic neurones: presynaptic mechanisms. J Neurochem. 2006;96:1540-50.

21. Oh SB, Tran PB, Gillard SE, Hurley RW, Hammond DL, Miller RJ. Chemokines and glycoprotein120 produce pain hypersensitivity by directly exciting primary nociceptive neurons. J Neurosci. 2001;21:5027-35.

22. Cummins TR, Sheets PL, Waxman SG. The roles of sodium channels in nociception: implications for mechanisms of pain. Pain. 2007;131:243-57.

23. Liu M, Wood JN. The roles of sodium channels in nociception: implications for mechanisms of neuropathic pain. Pain Med. 2011;12 Suppl 3:S93-9.

24. Dib-Hajj SD, Cummins TR, Black JA, Waxman SG. Sodium channels in normal and pathological pain. Annu Rev Neurosci. 2010;33:325-47.

25. Belkouch M, Dansereau MA, Tetreault P, Biet M, Beaudet N, Dumaine R, et al. Functional up-regulation of Nav1.8 sodium channel in Abeta afferent fibers subjected to chronic peripheral inflammation. J Neuroinflammation. 2014;11:45.

26. He XH, Zang Y, Chen X, Pang RP, Xu JT, Zhou X, et al. TNF-alpha contributes to up-regulation of Nav1.3 and Nav1.8 in DRG neurons following motor fiber injury. Pain. 2010;151:266-79.

27. Liang $L$, Fan $L$, Tao $B$, Yaster $M$, Tao YX. Protein kinase $B / A k t$ is required for complete Freund's adjuvant-induced upregulation of Nav1.7 and Nav1.8 in primary sensory neurons. J Pain. 2013;14:638-47.

28. Yu YQ, Zhao F, Guan SM, Chen J. Antisense-mediated knockdown of $\mathrm{Na}(\mathrm{V}) 1$. 8 , but not $\mathrm{Na}(\mathrm{V}) 1.9$, generates inhibitory effects on complete Freund's adjuvant-induced inflammatory pain in rat. PLOS One. 2011;6:e19865.

29. Yu YQ, Zhao ZY, Chen XF, Xie F, Yang Y, Chen J. Activation of tetrodotoxinresistant sodium channel NaV1.9 in rat primary sensory neurons contributes to melittin-induced pain behavior. Neuromolecular Med. 2013;15:209-17.

30. Kao DJ, Li AH, Chen JC, Luo RS, Chen YL, Lu JC, et al. CC chemokine ligand 2 upregulates the current density and expression of TRPV1 channels and Nav1.8 sodium channels in dorsal root ganglion neurons. J Neuroinflammation. 2012;9:189.

31. Wang JG, Strong JA, Xie W, Yang RH, Coyle DE, Wick DM, et al. The chemokine CXCL1/growth related oncogene increases sodium currents and neuronal excitability in small diameter sensory neurons. Mol Pain. 2008;4:38.

32. Chen J, Chen HS. Pivotal role of capsaicin-sensitive primary afferents in development of both heat and mechanical hyperalgesia induced by intraplantar bee venom injection. Pain. 2001;91:367-76.

33. Chen J, Lariviere WR. The nociceptive and anti-nociceptive effects of bee venom injection and therapy: a double-edged sword. Prog Neurobiol. 2010;92:151-83

34. Chen J, Luo C, Li H, Chen H. Primary hyperalgesia to mechanical and heat stimuli following subcutaneous bee venom injection into the plantar surface of hindpaw in the conscious rat: a comparative study with the formalin test. Pain. 1999:83:67-76.

35. $Y u Y Q, C h e n ~ J$. Activation of spinal extracellular signaling-regulated kinases by intraplantar melittin injection. Neurosci Lett. 2005;381:194-8.

36. Hao J, Liu MG, Yu YQ, Cao FL, Li Z, Lu ZM, et al. Roles of peripheral mitogen-activated protein kinases in melittin-induced nociception and hyperalgesia. Neuroscience. 2008;152:1067-75. 
37. Mert T, Gunes Y. Antinociceptive activities of lidocaine and the nav1.8 blocker a803467 in diabetic rats. J Am Assoc Lab Anim Sci. 2012;51:579-85.

38. Moon JY, Song S, Yoon SY, Roh DH, Kang SY, Park JH, et al. The differential effect of intrathecal Nav1.8 blockers on the induction and maintenance of capsaicin- and peripheral ischemia-induced mechanical allodynia and thermal hyperalgesia. Anesth Analg. 2012;114:215-23.

39. Wu WP, Xu XJ, Hao JX. Chronic lumbar catheterization of the spinal subarachnoid space in mice. J Neurosci Methods. 2004;133:65-9.

40. Du Y, Xiao Y, Lu ZM, Ding J, Xie F, Fu H, et al. Melittin activates TRPV1 receptors in primary nociceptive sensory neurons via the phospholipase A2 cascade pathways. Biochem Biophys Res Commun. 2011;408:32-7.

41. Yu YQ, Chen XF, Yang Y, Yang F, Chen J. Electrophysiological identification of tonic and phasic neurons in sensory dorsal root ganglion and their distinct implications in inflammatory pain. Physiol Res. 2014;63:793-9.

42. Floridi F, Trettel F, Di Bartolomeo S, Ciotti MT, Limatola C. Signalling pathways involved in the chemotactic activity of CXCL12 in cultured rat cerebellar neurons and CHP100 neuroepithelioma cells. J Neuroimmunol. 2003;135:38-46.

43. Luo Y, Lathia J, Mughal M, Mattson MP. SDF1alpha/CXCR4 signaling, via ERKs and the transcription factor Egr1, induces expression of a 67-kDa form of glutamic acid decarboxylase in embryonic hippocampal neurons. J Biol Chem. 2008;283:24789-800

44. Lv B, Yang X, Lv S, Wang L, Fan K, Shi R, et al. CXCR4 Signaling induced epithelial-mesenchymal transition by PI3K/AKT and ERK pathways in glioblastoma. Mol Neurobiol. 2014;52:1263-68.

45. Chen J, Luo C, Li HL. The contribution of spinal neuronal changes to development of prolonged, tonic nociceptive responses of the cat induced by subcutaneous bee venom injection. Eur J Pain. 1998;2:359-76.

46. Chen J, Li H, Luo C, Li Z, Zheng J. Involvement of peripheral NMDA and non-NMDA receptors in development of persistent firing of spinal widedynamic-range neurons induced by subcutaneous bee venom injection in the cat. Brain Res. 1999;844:98-105.

47. Chen J, Guan S. Bee venom and pain. In: Gopalakrishnakone P, editor. Toxinology: Toxins and Drug Discovery. Springer, in press.

48. Souza GR, Talbot J, Lotufo CM, Cunha FQ, Cunha TM, Ferreira SH. Fractalkine mediates inflammatory pain through activation of satellite glial cells. Proc Natl Acad Sci U S A. 2013;110:11193-8.

49. Bhangoo SK, Ren D, Miller RJ, Chan DM, Ripsch MS, Weiss C, et al. CXCR4 chemokine receptor signaling mediates pain hypersensitivity in association with antiretroviral toxic neuropathy. Brain Behav Immun. 2007:21:581-91.

50. Menichella DM, Abdelhak B, Ren D, Shum A, Frietag C, Miller RJ. CXCR4 chemokine receptor signaling mediates pain in diabetic neuropathy. Mol Pain. 2014;10:42

51. Wilson NM, Jung $H$, Ripsch MS, Miller RJ, White FA. CXCR4 signaling mediates morphine-induced tactile hyperalgesia. Brain Behav Immun. 2011;25:565-73.

52. White FA, Sun J, Waters SM, Ma C, Ren D, Ripsch M, et al. Excitatory monocyte chemoattractant protein-1 signaling is up-regulated in sensory neurons after chronic compression of the dorsal root ganglion. Proc Natl Acad Sci U S A. 2005;102:14092-7.

53. Zhang N, Inan S, Cowan A, Sun R, Wang JM, Rogers TJ, et al. A proinflammatory chemokine, CCL3, sensitizes the heat- and capsaicin-gated ion channel TRPV1. Proc Natl Acad Sci U S A. 2005:102:4536-41.

54. Bostock H, Rothwell JC. Latent addition in motor and sensory fibres of human peripheral nerve. J Physiol. 1997:498:277-94.

55. Nodera H, Kaji R. Nerve excitability testing and its clinical application to neuromuscular diseases. Clin Neurophysiol. 2006;117:1902-16.

56. Andres C, Hasenauer J, Ahn HS, Joseph EK, Isensee J, Theis FJ, et al. Woundhealing growth factor, basic FGF, induces Erk1/2-dependent mechanical hyperalgesia. Pain. 2013;154:2216-26.

57. Belkouch M, Dansereau MA, Reaux-Le GA, Van Steenwinckel J, Beaudet N, Chraibi A, et al. The chemokine CCL2 increases Nav1.8 sodium channel activity in primary sensory neurons through a Gbetagamma-dependent mechanism. J Neurosci. 2011;31:18381-90.

58. Zhao R, Pei GX, Cong R, Zhang H, Zang CW, Tian T. PKC-NF-kappaB are involved in CCL2-induced Nav1.8 expression and channel function in dorsal root ganglion neurons. Biosci Rep. 2014;34(3):pii: e00111.

59. Shen KF, Zhu HQ, Wei XH, Wang J, Li YY, Pang RP, et al. Interleukin-10 down-regulates voltage gated sodium channels in rat dorsal root ganglion neurons. Exp Neurol. 2013:247:466-75.
60. Akopian AN, Souslova V, England S, Okuse K, Ogata N, Ure J, et al. The tetrodotoxin-resistant sodium channel SNS has a specialized function in pain pathways. Nat Neurosci. 1999;2:541-8

61. Han C, Estacion M, Huang J, Vasylyev DV, Zhao P, Dib-Hajj S, et al. Human Nav1.8: enhanced persistent and ramp currents contribute to distinct firing properties of human DRG neurons. J Neurophysiol. 2015:113:3172-85.

62. Liang J, Liu X, Pan M, Dai W, Dong Z, Wang X, et al. Blockade of Nav1.8 currents in nociceptive trigeminal neurons contributes to antitrigeminovascular nociceptive effect of amitriptyline. Neuromolecular Med. 2014;16:308-21.

63. Abrahamsen B, Zhao J, Asante CO, Cendan CM, Marsh S, Martinez-Barbera $J P$, et al. The cell and molecular basis of mechanical, cold, and inflammatory pain. Science. 2008:321:702-5.

64. Zimmermann K, Leffler A, Babes A, Cendan CM, Carr RW, Kobayashi J, et al. Sensory neuron sodium channel Nav1.8 is essential for pain at low temperatures. Nature. 2007:447:855-8.

65. Jarvis MF, Honore $\mathrm{P}$, Shieh CC, Chapman M, Joshi S, Zhang XF, et al. A-803467, a potent and selective Nav1.8 sodium channel blocker, attenuates neuropathic and inflammatory pain in the rat. Proc Natl Acad Sci U S A. 2007;104:8520-5.

66. Dib-Hajj SD, Binshtok AM, Cummins TR, Jarvis MF, Samad T, Zimmermann K. Voltage-gated sodium channels in pain states: role in pathophysiology and targets for treatment. Brain Res Rev. 2009;60:65-83.

67. England S. Voltage-gated sodium channels: the search for subtype-selective analgesics. Expert Opin Investig Drugs. 2008;17:1849-64.

68. Dworkin RH, O'Connor AB, Audette J, Baron R, Gourlay GK, Haanpaa ML, et al. Recommendations for the pharmacological management of neuropathic pain: an overview and literature update. Mayo Clin Proc. 2010;85:S3-14.

\section{Submit your next manuscript to BioMed Central and we will help you at every step:}

- We accept pre-submission inquiries

- Our selector tool helps you to find the most relevant journal

- We provide round the clock customer support

- Convenient online submission

- Thorough peer review

- Inclusion in PubMed and all major indexing services

- Maximum visibility for your research

Submit your manuscript at www.biomedcentral.com/submit 